\title{
Characterization of graphs with exactly two non-negative eigenvalues
}

\author{
Mohammad Reza Oboudi * \\ Department of Mathematics, College of Sciences, Shiraz University, \\ Shiraz, 71457-44776, Iran \\ School of Mathematics, Institute for Research in Fundamental Sciences (IPM), \\ P.O. Box 19395-5746, Tehran, Iran
}

Received 28 March 2016, accepted 26 June 2016, published online 23 December 2016

\begin{abstract}
In this paper we characterize all graphs with exactly two non-negative eigenvalues. As a consequence we obtain all graphs $G$ such that $\lambda_{3}(G)<0$, where $\lambda_{3}(G)$ is the third largest eigenvalue of $G$.
\end{abstract}

Keywords: Spectrum and eigenvalues of graphs, graphs with exactly two non-negative eigenvalues.

Math. Subj. Class.: 05C31, 05C50, 05C75, 05C76, 15A18

\section{Introduction}

Throughout this paper all graphs are simple, that is finite and undirected without loops and multiple edges. Let $G$ be a graph with vertex set $\left\{v_{1}, \ldots, v_{n}\right\}$. The adjacency matrix of $G, A(G)=\left[a_{i j}\right]$, is an $n \times n$ matrix such that $a_{i j}=1$ if $v_{i}$ and $v_{j}$ are adjacent, and $a_{i j}=0$, otherwise. Thus $A(G)$ is a symmetric matrix with zeros on the diagonal and all the eigenvalues of $A(G)$ are real. By the eigenvalues of $G$ we mean those of its adjacency matrix. We denote the eigenvalues of $G$ by $\lambda_{1}(G) \geq \cdots \geq \lambda_{n}(G)$. By the spectrum of $G$ that is denoted by $\operatorname{Spec}(G)$, we mean the multiset of eigenvalues of $G$. The characteristic polynomial of $G, \operatorname{det}(\lambda I-A(G))$, is denoted by $P(G, \lambda)$. Studying the eigenvalues of graphs, the roots of characteristic polynomials of graphs, has always been of great interest to researchers, for instance see $[1,2,3,4,5,6,7,8,9,10]$ and the references therein.

It is well known that $\lambda_{1}(G)+\cdots+\lambda_{n}(G)=0$ and $\lambda_{1}^{2}(G)+\cdots+\lambda_{n}^{2}(G)=2 m$, where $m$ is the number of edges of $G$. Thus if $G$ has at least one edge, then $G$ has at least one positive eigenvalue. One of the attractive problems is the characterization of graphs with a

* This research was supported in part by a grant from IPM (No. 95050012).

E-mail address: mr_oboudi@yahoo.com (Mohammad Reza Oboudi) 
few non-zero eigenvalues. In [5] all bipartite graphs with at most six non-zero eigenvalues have been characterized. The another interesting problem is the characterization of graphs with a few positive eigenvalues. In [10] Smith characterized all graphs with exactly one positive eigenvalue. In fact, a graph has exactly one positive eigenvalue if and only if its non-isolated vertices form a complete multipartite graph. In [9] Petrović has studied the characterization of graphs with exactly two non-negative eigenvalues. In this paper with a different proof we state a new characterization of all graphs $G$ with exactly two nonnegative eigenvalues. In other words we find the graphs $G$ with $\lambda_{1}(G) \geq 0, \lambda_{2}(G) \geq 0$ and $\lambda_{3}(G)<0$.

For a graph $G, V(G)$ and $E(G)$ denote the vertex set and the edge set of $G$, respectively; $\bar{G}$ denotes the complement of $G$. The order of $G$ denotes the number of vertices of $G$. The closed neighborhood of a vertex $v$ of $G$ which is denoted by $N[v]$, is the set $\{u \in$ $V(G): u v \in E(G)\} \cup\{v\}$. For every vertex $v \in V(G)$, the degree of $v$ is the number of edges incident with $v$ and is denoted by $\operatorname{deg}_{G}(v)$ (for simplicity we use $\operatorname{deg}(v)$ instead of $\operatorname{deg}_{G}(v)$ ). By $\delta(G)$ we mean the minimum degree of vertices of $G$. A set $S \subseteq V(G)$ is an independent set if there is no edge between the vertices of $S$. The independence number of $G, \alpha(G)$, is the maximum cardinality of an independent set of $G$. For two graphs $G$ and $H$ with disjoint vertex sets, $G+H$ denotes the graph with the vertex set $V(G) \cup V(H)$ and the edge set $E(G) \cup E(H)$, i.e. the disjoint union of two graphs $G$ and $H$. In particular, $n G$ denotes the disjoint union of $n$ copies of $G$. The complete product (join) $G \vee H$ of graphs $G$ and $H$ is the graph obtained from $G+H$ by joining every vertex of $G$ with every vertex of $H$. For positive integers $n_{1}, \ldots, n_{\ell}, K_{n_{1}, \ldots, n_{\ell}}$ denotes the complete multipartite graph with $\ell$ parts of sizes $n_{1}, \ldots, n_{\ell}$. Let $K_{n}, n K_{1}=\overline{K_{n}}, C_{n}$ and $P_{n}$ be the complete graph, the null graph, the cycle and the path on $n$ vertices, respectively.

\section{The structure of graphs with exactly two positive eigenvalues}

In this section we obtain a characterization of graphs that have exactly two positive eigenvalues. We need the Interlacing Theorem.

Theorem 2.1. ([4, Theorem 9.1.1]) Let $G$ be a graph of order $n$ and $H$ be an induced subgraph of $G$ with order $m$. Suppose that $\lambda_{1}(G) \geq \cdots \geq \lambda_{n}(G)$ and $\lambda_{1}(H) \geq \cdots \geq$ $\lambda_{m}(H)$ are the eigenvalues of $G$ and $H$, respectively. Then for every $i, 1 \leq \bar{i} \leq m$, $\lambda_{i}(G) \geq \lambda_{i}(H) \geq \lambda_{n-m+i}(G)$.

Theorem 2.2. ([10], see also [3, Theorem 6.7]) A graph has exactly one positive eigenvalue if and only if its non-isolated vertices form a complete multipartite graph.

First we characterize all graphs with exactly one non-negative eigenvalue.

Theorem 2.3. Let $G$ be a graph of order $n \geq 2$ with eigenvalues $\lambda_{1} \geq \cdots \geq \lambda_{n}$. Then $\lambda_{2}<0$ if and only if $G \cong K_{n}$.

Proof. If $G \cong K_{n}$ and $n \geq 2$, then $\lambda_{2}=-1$. Now suppose that $\lambda_{2}<0$. We show that $G \cong K_{n}$. Suppose that $G \nsubseteq K_{n}$. Thus $2 K_{1}$ is an induced subgraph of $G$. So by Interlacing Theorem 2.1, $\lambda_{2} \geq \lambda_{2}\left(2 K_{1}\right)=0$, a contradiction. Hence $G \cong K_{n}$.

Lemma 2.4. Let $G$ be a graph of order $n \geq 3$ with eigenvalues $\lambda_{1} \geq \cdots \geq \lambda_{n}$. Suppose that $\lambda_{1} \geq 0, \lambda_{2} \geq 0$ and $\lambda_{3}<0$. Then the following hold: 
1. If $G$ is disconnected, then $G \cong K_{r}+K_{n-r}$, for some positive integer $r$, where $r \leq n-1$.

2. If $G$ is connected and $\lambda_{2}=0$, then $G \cong K_{n} \backslash$ e for an edge e of $K_{n}$.

Proof. 1. Let $G$ be disconnected. Assume that $G_{1}, \ldots, G_{k}$ are the connected components of $G$, where $k \geq 2$. Since $\lambda_{1}\left(G_{1}\right) \geq 0, \ldots, \lambda_{1}\left(G_{k}\right) \geq 0$ are $k$ eigenvalues of $G$ and $\lambda_{3}<0$ we obtain that $k=2$. In other words $G$ has exactly two connected components. Thus $G=G_{1}+G_{2}$. We prove that $G_{1}$ and $G_{2}$ are complete graphs. First we show that $G_{1}$ is a complete graph. If $G_{1} \cong K_{1}$, there is nothing to prove. Assume that $\left|V\left(G_{1}\right)\right| \geq 2$ (equivalently $\left.G_{1} \not K_{1}\right)$. We claim that $\lambda_{2}\left(G_{1}\right)<0$. By contradiction suppose that $\lambda_{2}\left(G_{1}\right) \geq 0$. Since $\lambda_{1}\left(G_{1}\right) \geq 0, \lambda_{2}\left(G_{1}\right) \geq 0$ and $\lambda_{1}\left(G_{2}\right) \geq 0$ are three eigenvalues of $G$ we obtain that $\lambda_{3} \geq 0$, a contradiction (since $\lambda_{3}<0$ ). Hence the claim is proved. In other words $\lambda_{2}\left(G_{1}\right)<0$. So by Theorem $2.3, G_{1}$ is a complete graph. Similarly we obtain that $G_{2}$ is a complete graph. Hence $G$ is a disjoint union of two complete graphs.

2. Suppose that $G$ is connected and $\lambda_{2}=0$. Since $\lambda_{3}<0, G \neq n K_{1}$. Thus $\lambda_{1}>0$. Hence $G$ has exactly one positive eigenvalue. By Theorem 2.2 there are some positive integers $t$ and $n_{1} \geq \cdots \geq n_{t} \geq 1$, so that $n_{1}+\cdots+n_{t}=n$ and $G \cong K_{n_{1}, \ldots, n_{t}}$. If $t=1$, then $G \cong n K_{1}$, a contradiction (since $G$ is connected). Thus $t \geq 2$. If $n_{1}=1$, then $G \cong K_{n}$ and so $\lambda_{2}=-1$, a contradiction. Therefore $n_{1} \geq 2$. If $n_{2} \geq 2$, then $C_{4}$ is an induced subgraph of $G$. Using Interlacing Theorem 2.1 we get $\lambda_{3} \geq \lambda_{3}\left(C_{4}\right)=0$, a contradiction. Thus $n_{2}=\cdots=n_{t}=1$. Now if $n_{1} \geq 3$, then $K_{1,3}$ is an induced subgraph of $G$. Similarly by Interlacing Theorem 2.1 we obtain $\lambda_{3} \geq \lambda_{3}\left(K_{1,3}\right)=0$, a contradiction. So $n_{1}=2$. Thus $G \cong K_{2,1, \ldots, 1}$. In other words $G \cong K_{n} \backslash e$, for an edge $e$ of $K_{n}$. We note that

$$
\operatorname{Spec}\left(K_{n} \backslash e\right)=\{\frac{n-3+\sqrt{n^{2}+2 n-7}}{2}, 0, \underbrace{-1, \ldots,-1}_{n-3}, \frac{n-3-\sqrt{n^{2}+2 n-7}}{2}\} .
$$

The proof is complete.

In [2] all graphs $G$ with $\lambda_{1}>0, \lambda_{2} \leq 0$ and $\lambda_{3}<0$ have been characterized.

Remark 2.5. Let $n_{1}, \ldots, n_{t}$ be some positive integers and $G=K_{n_{1}, \ldots, n_{t}}$. Similar to the proof of the second part of Lemma 2.4 by Interlacing Theorem 2.1 one can see that $\lambda_{2}(G)<0$ if and only if $n_{1}=\cdots=n_{t}=1$. On the other hand by Theorem 2.2, $\lambda_{2}\left(K_{n_{1}, \ldots, n_{t}}\right) \leq 0$. Thus $\lambda_{2}\left(K_{n_{1}, \ldots, n_{t}}\right)=0$ if and only if $n_{k}>1$ for some $k$. In other words, the second largest eigenvalue of any complete multipartite graph except complete graph is zero.

Remark 2.6. Let $G$ be a graph of order $n \geq 3$ with eigenvalues $\lambda_{1} \geq \cdots \geq \lambda_{n}$. Assume that $G$ has exactly two non-negative eigenvalues. In other words, $\lambda_{1} \geq 0, \lambda_{2} \geq 0$ and $\lambda_{3}<0$. Since $\lambda_{3}<0, G \nsucceq n K_{1}$. Thus $\lambda_{1}>0$. Hence $\lambda_{1}>0, \lambda_{2} \geq 0$ and $\lambda_{3}<0$. If $G$ is disconnected, then by the first part of Lemma 2.4, $G \cong K_{r}+K_{n-r}$ for some positive integer $r \leq n-1$. If $G$ is connected and $\lambda_{2}=0$, then by the second part of Lemma 2.4, $G \cong K_{n} \backslash e$, where $e$ is an edge of $K_{n}$. Thus to characterize all graphs with exactly two non-negative eigenvalues it remains to find connected graphs $G$ such that $\lambda_{1}(G)>0$, $\lambda_{2}(G)>0$ and $\lambda_{3}(G)<0$. In sequel we find this characterization. 
Definition 2.7. A graph $G$ is called semi-complete if $G$ is a disjoint union of two complete graphs or is obtained by adding some new edges to disjoint union of two complete graphs (see Figure 1).

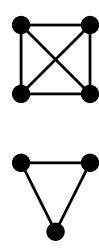

$H_{1}$

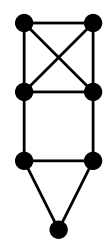

$H_{2}$

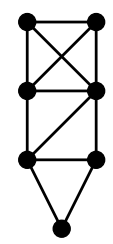

$H_{3}$

Figure 1: The graphs $H_{1}, H_{2}$ and $H_{3}$ are semi-complete that are obtained from $K_{3}+K_{4}$.

Now we prove one of the main results of this section.

Lemma 2.8. Let $G$ be a connected graph of order $n \geq 3$ and with eigenvalues $\lambda_{1} \geq \cdots \geq$ $\lambda_{n}$. If $\lambda_{2}>0$ and $\lambda_{3}<0$, then for every vertex $v \in V(G)$ with degree $\delta(G)$ we have $N[v] \cong K_{\delta(G)+1}$ and $G \backslash N[v] \cong K_{n-\delta(G)-1}$. In particular, $G$ is semi-complete.

Proof. Let $\lambda_{2}>0$ and $\lambda_{3}<0$. Since $\lambda_{2}>0, G$ is not complete graph. Therefore $\alpha(G) \geq 2$. If $\alpha(G) \geq 3$, then $3 K_{1}$ is an induced subgraph of $G$. Thus by Interlacing Theorem 2.1, $\lambda_{3}(G) \geq \lambda_{3}\left(3 K_{1}\right)=0$, a contradiction. Therefore $\alpha(G)=2$. Thus for every vertex $u \in V(G), G \backslash N[u]$ is a complete graph. In fact, $G \backslash N[u] \cong K_{n-\operatorname{deg}(u)-1}$.

Let $v_{0}$ be a vertex of $G$ with degree $\delta(G)$, that is $v_{0}$ has the minimum degree among all vertices of $G$. Since $G \nsubseteq K_{n}, \operatorname{deg}\left(v_{0}\right) \leq n-2$. Since $G \backslash N\left[v_{0}\right]$ is a complete graph, to complete the proof it is sufficient to show that the induced subgraph on the set $N\left[v_{0}\right]$ is a complete graph, that is every two vertices of $N\left[v_{0}\right]$ are adjacent. This also shows that $G$ is obtained by adding some edges to the complete graphs $N\left[v_{0}\right]$ and $G \backslash N\left[v_{0}\right]$ and so $G$ is semi-complete.

Now we show that $N\left[v_{0}\right]$ is a complete graph. By contradiction, suppose that $w$ and $z$ are two non-adjacent vertices of $N\left[v_{0}\right]$. Let $a$ be an arbitrary vertex of $V(G) \backslash N\left[v_{0}\right]$. The induced subgraph on $\left\{v_{0}, w, z, a\right\}$ in $G$ is one of the graphs, $A_{1}, A_{2}, A_{3}$ or $A_{4}$ (see Figure 2). Since $\lambda_{3}\left(A_{1}\right)=\lambda_{3}\left(A_{4}\right)=0$ and $\lambda_{3}<0$, Interlacing Theorem 2.1 shows that

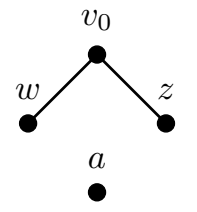

$A_{1}$

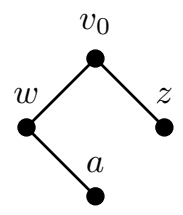

$A_{2}$

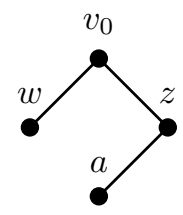

$A_{3}$

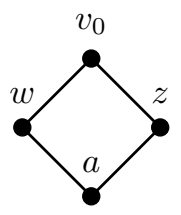

$A_{4}$

Figure 2: The subgraphs $A_{1}, A_{2}, A_{3}$ and $A_{4}$.

the induced subgraph on $\left\{v_{0}, w, z, a\right\}$ is $A_{2}$ or $A_{3}$. In other words any vertex of $G \backslash N\left[v_{0}\right]$ 
has exactly one neighbor in $\{w, z\}$. Without losing the generality assume that $a$ is adjacent to $w$. Now we show that every vertex of $G \backslash N\left[v_{0}\right]$ is adjacent to $w$. By contradiction suppose that $b \neq a$ is a vertex of $G \backslash N\left[v_{0}\right]$ such that $b$ is adjacent to $z$. Since $G \backslash N\left[v_{0}\right]$ is complete and $a, b \in V(G) \backslash N\left[v_{0}\right]$, the vertices $a$ and $b$ are adjacent. Thus the induced subgraph on $\left\{v_{0}, w, z, a, b\right\}$ is isomorphic to the cycle $C_{5}$. Since $\lambda_{3}\left(C_{5}\right) \simeq .618>0$, by Interlacing Theorem 2.1, we have $\lambda_{3}>0$, a contradiction. This contradiction shows that all vertices of $G \backslash N\left[v_{0}\right]$ are adjacent only to $w$. This implies that $\operatorname{deg}(z) \leq \operatorname{deg}\left(v_{0}\right)-1$, a contradiction, since $v_{0}$ has minimum degree. This contradiction completes the proof.

Claim 2.9. Let $G$ be a connected graph of order $n \geq 3$ and with eigenvalues $\lambda_{1} \geq \cdots \geq$ $\lambda_{n}$ such that $\lambda_{2}>0$ and $\lambda_{3}<0$. Let $X=N\left[v_{0}\right]$ and $Y=G \backslash N\left[v_{0}\right]$, where $v_{0}$ is a vertex of $G$ with degree $\delta(G)$. Then for every two vertices $a$ and $b$ in $X$ (also for $a$ and $b$ in $Y$ ) $N[a] \subseteq N[b]$ or $N[b] \subseteq N[a]$.

Proof. Let $a$ and $b$ be two vertices of $X$. We show that $N[a] \subseteq N[b]$ or $N[b] \subseteq N[a]$. First note that by Lemma $2.8, X$ is a complete graph. This implies that $N[a] \cap X=$ $N[b] \cap X=X$. Now by contradiction suppose that there are some vertices $c$ and $d$ in $Y$ such that $c \in N[a] \backslash N[b]$ and $d \in N[b] \backslash N[a]$. Thus the induced subgraph on $\{a, b, c, d\}$ is isomorphic to $C_{4}$. Using Interlacing Theorem 2.1 we get $\lambda_{3} \geq \lambda_{3}\left(C_{4}\right)=0$, a contradiction (since $\lambda_{3}<0$ ). Thus the result follows. Similarly one can prove that for any two vertices $v$ and $w$ in $Y, N[v] \subseteq N[w]$ or $N[w] \subseteq N[v]$.

As an example we find an infinite family of connected graphs with positive second largest eigenvalue and negative third largest eigenvalue.

Corollary 2.10. Let $n \geq 4$ be an integer. Let $K(n, t)$ be the graph obtained by deleting $t$ edges incident to one vertex of $K_{n}$, where $2 \leq t \leq n-2$. Then $\lambda_{2}(K(n, t))>0$ and $\lambda_{3}(K(n, t))<0$.

Proof. Let $\lambda_{1} \geq \cdots \geq \lambda_{n}$ be the eigenvalues of $K(n, t)$. Since $K_{n-1}$ is an induced subgraph of $K(n, t)$, by Interlacing Theorem $2.1, \lambda_{1} \geq n-2 \geq \lambda_{2} \geq-1 \geq \lambda_{3}$. Thus $\lambda_{3}<0$. On the other, since $K(n, t)$ is not a complete multipartite, by Theorem 2.2, $\lambda_{2}>0$.

Definition 2.11. A graph $G$ is called quasi-reduced if for every two vertices $u$ and $v$ of $G$, $N[u] \neq N[v]$.

As an example of quasi-reduced graphs, we define the graphs $G_{n}$ that have important role for characterizing graphs with $\lambda_{2}>0$ and $\lambda_{3}<0$.

Definition 2.12. For every integer $n \geq 2$, let $G_{n}$ be the graph of order $n$ such that $G_{n}$ is obtained from disjoint complete graphs $K_{\left\lceil\frac{n}{2}\right\rceil}$ and $K_{\left\lfloor\frac{n}{2}\right\rfloor}$ as following: Let $V\left(K_{\left\lceil\frac{n}{2}\right\rceil}\right)=$ $\left\{v_{1}, \ldots, v_{\left\lceil\frac{n}{2}\right\rceil}\right\}$ and $V\left(K_{\left\lfloor\frac{n}{2}\right\rfloor}\right)=\left\{w_{1}, \ldots, w_{\left\lfloor\frac{n}{2}\right\rfloor}\right\}$. Then add some new edges to $K_{\left\lceil\frac{n}{2}\right\rceil}+$ $K_{\left\lfloor\frac{n}{2}\right\rfloor}$ such that the following hold:

(i) $N\left[v_{1}\right] \subset \cdots \subset N\left[v_{\left\lceil\frac{n}{2}\right\rceil}\right]$ and $N\left[w_{1}\right] \subset \cdots \subset N\left[w_{\left\lfloor\frac{n}{2}\right\rfloor}\right]$.

(ii) $\left|N\left[v_{i}\right] \cap V\left(K_{\left\lfloor\frac{n}{2}\right\rfloor}\right)\right|=i-1$ for $i=1, \ldots,\left\lceil\frac{n}{2}\right\rceil$.

(iii) $\left|N\left[w_{j}\right] \cap V\left(K_{\left\lceil\frac{n}{2}\right\rceil}\right)\right|=\left\{\begin{array}{ll}j-1, & \text { if } n \text { is even; } \\ j, & \text { if } n \text { is odd }\end{array}\right.$ for $j=1, \ldots,\left\lfloor\frac{n}{2}\right\rfloor$. 
In Figure 3, the graphs $G_{2}, G_{3}, G_{4}, G_{5}$ and $G_{6}$ have been shown. In addition in Figure 4 one can see the complement of $G_{7}, \ldots, G_{12}$. We note that $G_{2 k}=B_{2 k}(1, \ldots, 1 ; 1, \ldots, 1)$ and $G_{2 k+1}=B_{2 k+1}(1, \ldots, 1 ; 1, \ldots, 1 ; 1)$, where $B_{2 k}$ and $B_{2 k+1}$ are the graphs that have been defined in [9].

Remark 2.13. For every $n \geq 2, G_{n}$ is semi-complete and quasi-reduced. In addition if $n \geq$ 3 , then $G_{n}$ is connected. We note that for every $n \geq 3, G_{n}$ is an induced subgraph of $G_{n+1}$. In fact if $n$ is even, then $G_{n+1} \cong K_{1} \vee G_{n}$ and if $n$ is odd, then $G_{n+1}$ is obtained from $G_{n}$ by adding a new vertex $w$ such that $w$ is adjacent to any vertex of $\left\{w_{1}, \ldots, w_{\left\lfloor\frac{n}{2}\right\rfloor}\right\}=$ $V\left(K_{\left\lfloor\frac{n}{2}\right\rfloor}\right)$, where $K_{\left\lfloor\frac{n}{2}\right\rfloor}$ is one of the parts of $G_{n}$ (see Definition 2.12).

Remark 2.14. We note that for every $n \geq 2$, the group of all automorphisms of the graph $G_{n}, \operatorname{Aut}\left(G_{n}\right)$, has exactly two elements.

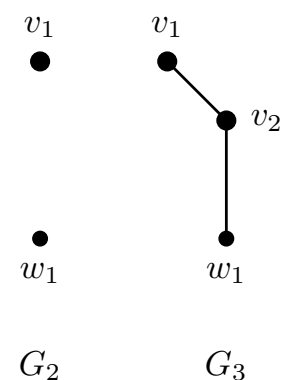

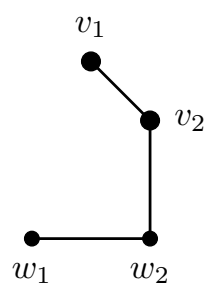

$G_{4}$

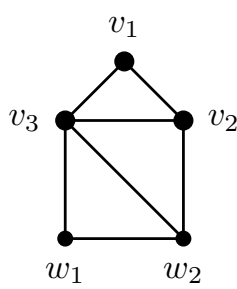

$G_{5}$

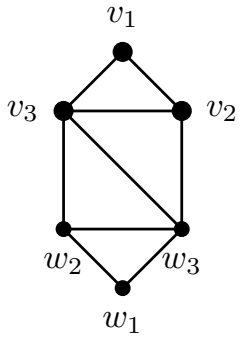

$G_{6}$

Figure 3: The graphs $G_{2}, G_{3}, G_{4}, G_{5}$ and $G_{6}$ are semi-complete and quasi-reduced.

The next result shows that there is only one connected quasi-reduced graph with $\lambda_{2}>0$ and $\lambda_{3}<0$.

Lemma 2.15. Let $G$ be a connected graph of order $n \geq 3$. If $G$ is quasi-reduced and $\lambda_{2}(G)>0$ and $\lambda_{3}(G)<0$, then $G \cong G_{n}$.

Proof. Assume that $\lambda_{2}(G)>0$ and $\lambda_{3}(G)<0$. Since $G$ is connected, by Lemma 2.8, $G$ is semi-complete. Let $\delta(G)=t$ and $v_{1}^{\prime}$ be a vertex of $G$ with degree $t$. By Lemma 2.8, $N\left[v_{1}^{\prime}\right] \cong K_{t+1}$ and $G \backslash N\left[v_{1}^{\prime}\right] \cong K_{n-t-1}$. In fact $G$ is obtained from the disjoint complete graphs $K_{t+1}$ and $K_{n-t-1}$ by adding some new edges (see the proof of Lemma 2.8). Let $V\left(K_{t+1}\right)=\left\{v_{1}^{\prime}, v_{2}^{\prime}, \ldots, v_{t+1}^{\prime}\right\}$ and $V\left(K_{n-t-1}\right)=\left\{w_{1}^{\prime}, \ldots, w_{n-t-1}^{\prime}\right\}$. By Claim 2.9, for every two vertices $v_{i}^{\prime}$ and $v_{j}^{\prime}$ in $K_{t+1}, N\left[v_{i}^{\prime}\right] \subseteq N\left[v_{j}^{\prime}\right]$ or $N\left[v_{j}^{\prime}\right] \subseteq N\left[v_{i}^{\prime}\right]$. Also for every two vertices $w_{i}^{\prime}$ and $w_{j}^{\prime}$ in $K_{n-t-1}, N\left[w_{i}^{\prime}\right] \subseteq N\left[w_{j}^{\prime}\right]$ or $N\left[w_{j}^{\prime}\right] \subseteq N\left[w_{i}^{\prime}\right]$. So without losing the generality assume that $N\left[v_{1}^{\prime}\right] \subseteq \cdots \subseteq N\left[v_{t+1}^{\prime}\right]$ and $N\left[w_{1}^{\prime}\right] \subseteq \cdots \subseteq N\left[w_{n-t-1}^{\prime}\right]$ (note that $N\left[v_{1}^{\prime}\right]=V\left(K_{t+1}\right)$ and for every $\left.1 \leq i \leq t+1, N\left[v_{1}^{\prime}\right] \subseteq N\left[v_{i}^{\prime}\right]\right)$. Now suppose that $G$ is quasi-reduced. Therefore we find that

$$
0=\left|N\left[v_{1}^{\prime}\right] \cap V\left(K_{n-t-1}\right)\right|<\cdots<\left|N\left[v_{t+1}^{\prime}\right] \cap V\left(K_{n-t-1}\right)\right| \leq n-t-1,
$$

and

$$
0 \leq\left|N\left[w_{1}^{\prime}\right] \cap V\left(K_{t+1}\right)\right|<\cdots<\left|N\left[w_{n-t-1}^{\prime}\right] \cap V\left(K_{t+1}\right)\right| \leq t
$$



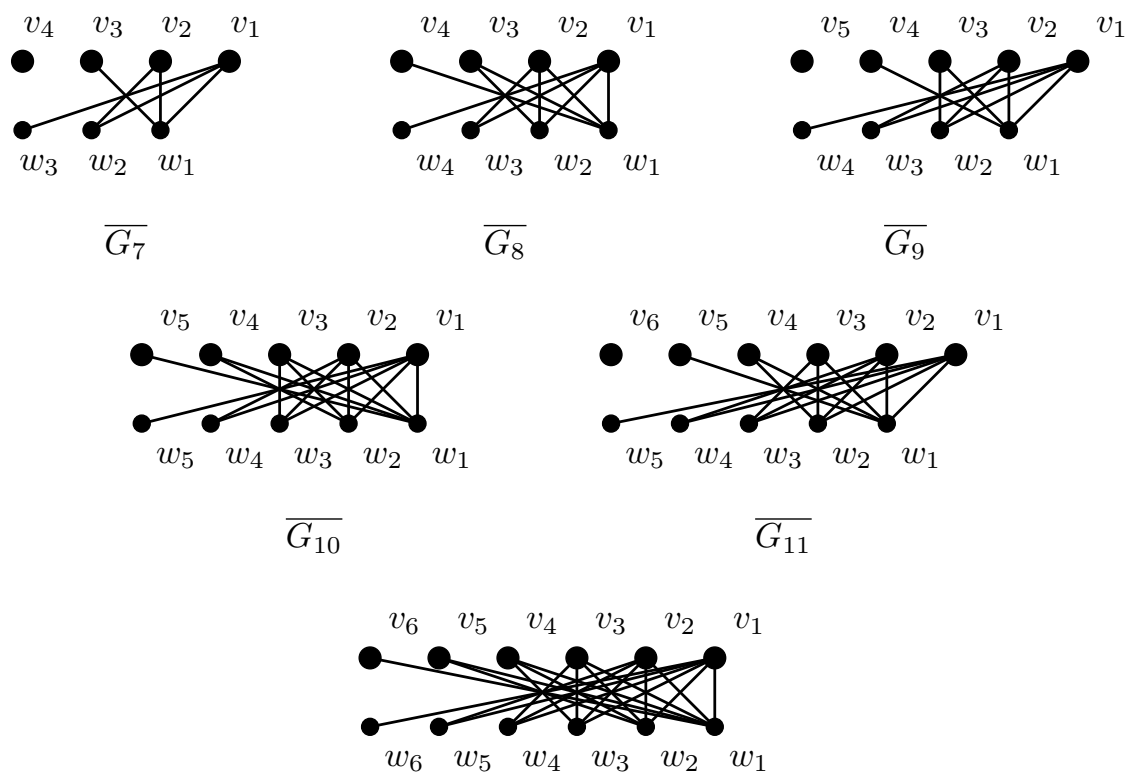

$$
\overline{G_{12}}
$$

Figure 4: The complement graphs of $G_{7}, G_{8}, G_{9}, G_{10}, G_{11}$ and $G_{12}$.

Since $\left|N\left[v_{1}^{\prime}\right] \cap V\left(K_{n-t-1}\right)\right|, \ldots,\left|N\left[v_{t+1}^{\prime}\right] \cap V\left(K_{n-t-1}\right)\right|$ are $t+1$ distinct integers between 0 and $n-t-1$, the Equation (2.1) shows that $t \leq n-t-1$. Similarly, the Equation (2.2) implies that $n-t-2 \leq t$. Hence $n-2 \leq 2 t \leq n-1$. So $t=\left\lceil\frac{n}{2}\right\rceil-1$.

If $n$ is even, then the Equation (2.2) shows that $\left|N\left[w_{j}^{\prime}\right] \cap V\left(K_{t+1}\right)\right|=j-1$, for $j=1, \ldots, n-t-1$. So $w_{1}^{\prime}$ has no neighbor in $K_{t+1}$. Thus for any $1 \leq i \leq t+1$, $\left|N\left[v_{i}^{\prime}\right] \cap V\left(K_{n-t-1}\right)\right| \leq n-t-2$. Using Equation (2.1) we conclude that $\mid N\left[v_{i}^{\prime}\right] \cap$ $V\left(K_{n-t-1}\right) \mid=i-1$, for $i=1, \ldots, t+1$. Hence $G \cong G_{n}$.

Similarly, for odd $n$ we obtain that $\left|N\left[v_{i}^{\prime}\right] \cap V\left(K_{n-t-1}\right)\right|=i-1$, for $i=1, \ldots, t+1$. Thus $v_{t+1}^{\prime}$ is adjacent to every vertex of $V\left(K_{n-t-1}\right)$. Hence $1 \leq\left|N\left[w_{1}^{\prime}\right] \cap V\left(K_{t+1}\right)\right|$. Using inequality (2.2) we find that $\left|N\left[w_{j}^{\prime}\right] \cap V\left(K_{t+1}\right)\right|=j$, for $j=1, \ldots, n-t-1$. Thus $G \cong G_{n}$.

Lemma 2.16. Let $G_{n}$ be the semi-complete and quasi-reduced graph as mentioned above. Then $\lambda_{2}\left(G_{n}\right)>0$ and $\lambda_{3}\left(G_{n}\right)<0$ if and only if $4 \leq n \leq 12$.

Proof. One can see that $\lambda_{2}\left(G_{3}\right)=0$ and for every $4 \leq n \leq 12, \lambda_{2}\left(G_{n}\right)>0$ and $\lambda_{3}\left(G_{n}\right)<0$. Now assume that $n \geq 13$. Since $\lambda_{3}\left(G_{13}\right)=0$ and $G_{13}$ is an induced subgraph of $G_{n}$ (by Remark 2.13), by Interlacing Theorem 2.1 we find that $\lambda_{3}\left(G_{n}\right) \geq$ $\lambda_{3}\left(G_{13}\right)=0$. This completes the proof.

Definition 2.17. Let $G$ be a graph with vertex set $\left\{v_{1}, \ldots, v_{n}\right\}$. By $G\left[K_{t_{1}}, \ldots, K_{t_{n}}\right]$ we mean the graph obtained by replacing the vertex $v_{j}$ by the complete graph $K_{t_{j}}$ for 
$1 \leq j \leq n$, where every vertex of $K_{t_{i}}$ is adjacent to every vertex of $K_{t_{j}}$ if and only if $v_{i}$ is adjacent to $v_{j}$ (in $G$ ). For example $K_{2}\left[K_{p}, K_{q}\right] \cong K_{p+q}$ and $\overline{K_{2}}\left[K_{p}, K_{q}\right] \cong K_{p}+K_{q}$.

Now we prove one of the main results of the paper.

Theorem 2.18. Let $G$ be a connected graph of order $n \geq 3$. If $\lambda_{2}(G)>0$ and $\lambda_{3}(G)<0$, then there exist some positive integers $s$ and $t_{1}, \ldots, t_{s}$ so that $3 \leq s \leq 12$ and $t_{1}+\cdots+t_{s}=$ $n$ and $G \cong G_{s}\left[K_{t_{1}}, \ldots, K_{t_{s}}\right]$.

Proof. Suppose that $\lambda_{2}(G)>0$ and $\lambda_{3}(G)<0$. By Lemma 2.8, $G$ is semi-complete. If $G$ is quasi-reduced, then by Lemma $2.15, G \cong G_{n} \cong G_{n}\left[K_{1}, \ldots, K_{1}\right]$. So $\lambda_{2}\left(G_{n}\right)=$ $\lambda_{2}(G)>0$ and $\lambda_{3}\left(G_{n}\right)=\lambda_{3}(G)<0$. Hence by Lemma 2.16, $4 \leq n \leq 12$.

Now assume that $G$ is not quasi-reduced. Thus there exists a connected induced subgraph of $G$, say $H$, such that $H$ is quasi-reduced and $G=H\left[K_{t_{1}}, \ldots, K_{t_{s}}\right]$, where $s$ is the order of $H$ and $t_{1}, \ldots, t_{s}$ are some positive integers. Thus $t_{1}+\cdots+t_{s}=n$. If $H \cong K_{s}$, then $G \cong K_{n}$, a contradiction (since $\lambda_{2}\left(K_{n}\right)=-1<0$ while $\lambda_{2}(G)>0$ ). Thus $H$ is not a complete graph. On the other hand $H$ is a connected graph of order $s$. Thus $s \geq 3$. Since $H$ is obtained from $G$ by removing some vertices and $G$ is semi-complete, $H$ is also semi-complete. Suppose that $C_{4}$ is an induced subgraph of $H$. Since $H$ is an induced subgraph of $G$, by Interlacing Theorem 2.1 we conclude that $\lambda_{3}(G) \geq \lambda_{3}(H) \geq \lambda_{3}\left(C_{4}\right)=0$, a contradiction. Thus $H$ has no induced cycle $C_{4}$.

Now we show that $H \cong G_{s}$. Since $H$ is semi-complete, $H$ is obtained from the disjoint union of two complete graphs, say $K_{p}$ and $K_{q}$, for some positive integers $p$ and $q$. Let $X=K_{p}$ and $Y=K_{q}$. We claim that for every two vertices $a, b \in V(X), N[a] \subseteq N[b]$ or $N[b] \subseteq N[a]$. By contradiction assume that $N[a] \nsubseteq N[b]$ and $N[b] \nsubseteq N[a]$. Thus there are two vertices $c$ and $d$ such that $c \in N[a] \backslash N[b]$ and $d \in N[b] \backslash N[a]$. Since $V(X) \subseteq N[a] \cap N[b]$, we find that $c$ and $d$ are two vertices of $Y$. Now we remark that the induced subgraph on the vertices $a, b, c, d$ is isomorphic to $C_{4}$. It is a contradiction, since $H$ has no induced cycle $C_{4}$. So the claim holds. Similarly for every two vertices $z, w \in V(Y), N[z] \subseteq N[w]$ or $N[w] \subseteq N[z]$. On the other hand $H$ is quasi-reduced, thus similar to the proof of Lemma 2.15 one can see that $H \cong G_{s}$.

If $s \geq 13$, then by Remark 2.13, $G_{13}$ is an induced subgraph of $H$ and so is an induced subgraph of $G$. Thus by Interlacing Theorem $2.1, \lambda_{3}(G) \geq \lambda_{3}\left(G_{13}\right)=0$, a contradiction, since $\lambda_{3}(G)<0$. Hence $s \leq 12$. The proof is complete.

We end this section by characterization the graphs with $\lambda_{3}<0$. We note that if $G$ is a graph with $\lambda_{3}(G)<0$, then $G$ is not the null graph. Thus $\lambda_{1}(G)>0$. Using Remark 2.5, the second part of Lemma 2.4 and Theorems 2.2, 2.3 and 2.18 we obtain this characterization.

Theorem 2.19. Let $G$ be a graph with eigenvalues $\lambda_{1} \geq \cdots \geq \lambda_{n}$. Assume that $\lambda_{3}<0$. Then the following hold:

1. If $\lambda_{1}>0$ and $\lambda_{2}>0$, then $G \cong K_{p}+K_{q}$ for some integers $p, q \geq 2$ or there exist some positive integers $s$ and $t_{1}, \ldots, t_{s}$ so that $3 \leq s \leq 12$ and $t_{1}+\cdots+t_{s}=n$ and $G \cong G_{s}\left[K_{t_{1}}, \ldots, K_{t_{s}}\right]$.

2. If $\lambda_{1}>0$ and $\lambda_{2}=0$, then $G \cong K_{1}+K_{n-1}$ or $G \cong K_{n} \backslash e$, where $e$ is an edge of $K_{n}$.

3. If $\lambda_{1}>0$ and $\lambda_{2}<0$, then $G \cong K_{n}$. 
Since $K_{n} \backslash e \cong G_{3}\left[K_{1}, K_{1}, K_{n-2}\right]$ and $K_{p}+K_{q} \cong \overline{K_{2}}\left[K_{p}, K_{q}\right]$, we can rewrite Theorem 2.19 as following:

Theorem 2.20. Let $G$ be a graph. If $\lambda_{3}(G)<0$, then $G \cong K_{n}$ or there exist some positive integers $s$ and $t_{1}, \ldots, t_{s}$ such that $2 \leq s \leq 12$ and $t_{1}+\cdots+t_{s}=n$ and $G \cong G_{s}\left[K_{t_{1}}, \ldots, K_{t_{s}}\right]$.

In the next section we investigate the converse of Theorem 2.20. In other words we obtain all values of $t_{1}, \ldots, t_{s}$ (for $2 \leq s \leq 12$ ) such that $\lambda_{3}\left(G_{s}\left[K_{t_{1}}, \ldots, K_{t_{s}}\right]\right)<0$. We need the following important result for computing the characteristic polynomial of $G_{s}\left[K_{t_{1}}, \ldots, K_{t_{s}}\right]$, the polynomial $P\left(G_{n}\left[K_{t_{1}}, \ldots, K_{t_{n}}\right], \lambda\right)$.

Theorem 2.21. [7] Let $n \geq 2$. Suppose that $\left\{v_{1}, \ldots, v_{n}\right\}$ is the vertex set of $G_{n}$ and $A=\left[a_{i j}\right]$ is the adjacency matrix of $G_{n}$ with respect to $\left\{v_{1}, \ldots, v_{n}\right\}\left(a_{i j}=1\right.$ if and only if $v_{i}$ and $v_{j}$ are adjacent and $a_{i j}=0$, otherwise). Let $t_{1}, \ldots, t_{n}$ be some positive integers and $M=\left[m_{i j}\right]$ be a $n \times n$ matrix, where

$$
m_{i j}:= \begin{cases}t_{i}-1, & \text { if } i=j \\ a_{i j} t_{j}, & \text { if } i \neq j .\end{cases}
$$

Then

$$
P\left(G_{n}\left[K_{t_{1}}, \ldots, K_{t_{n}}\right], \lambda\right)=(\lambda+1)^{t_{1}+\cdots+t_{n}-n} g(\lambda),
$$

where $g(\lambda)=\operatorname{det}(\lambda I-M)$. In addition, the multiplicity of -1 as an eigenvalue of $G_{n}\left[K_{t_{1}}, \ldots, K_{t_{n}}\right]$ is equal to $t_{1}+\cdots+t_{n}-n$.

\section{The list of all connected graphs with $\lambda_{2}>0$ and $\lambda_{3}<0$}

In this section we investigate the converse of Theorem 2.20. We use Petrović's notation [9] that is very similar to the notation of Definition 2.17. We note that in Definition 2.17, the graph $G\left[H_{1}, \ldots, H_{n}\right]$ is dependent to the labeling of the vertices of $G$ while in the next definition first we fix a labeling for the vertices of $G_{n}$ (see Definition 2.12), and then use the operation of Definition 2.17. For instance we consider the labeling $v_{1}, \ldots, v_{s}$ and $w_{1}, \ldots, w_{s}$ for the vertices of $G_{2 s}$ and then apply the operation of Definition 2.17.

Definition 3.1. Let $s \geq 1$ be an integer and $n_{1}, \ldots, n_{2 s+1}$ be some positive integers. Let $B_{2 s}\left(n_{1}, \ldots, n_{s} ; n_{s+1}, \ldots, n_{2 s}\right)$ denote the graph obtained from $G_{2 s}$ by replacing the vertices $v_{1}$ by $K_{n_{1}}, v_{2}$ by $K_{n_{2}}, \ldots$, and $v_{s}$ by $K_{n_{s}}$ and $w_{1}$ by $K_{n_{s+1}}, w_{2}$ by $K_{n_{s+2}}, \ldots$, and $w_{s}$ by $K_{n_{2 s}}$ (see Definition 2.12). In other words

$$
B_{2 s}\left(n_{1}, \ldots, n_{s} ; n_{s+1}, \ldots, n_{2 s}\right)=G_{2 s}\left[K_{n_{1}}, \ldots, K_{n_{2 s}}\right],
$$

where the ordering of the vertices of $G_{2 s}$ is $V\left(G_{2 s}\right)=\left\{v_{1}, \ldots, v_{s}, w_{1}, \ldots, w_{s}\right\}$.

Similarly, by $B_{2 s+1}\left(n_{1}, \ldots, n_{s} ; n_{s+1}, \ldots, n_{2 s} ; n_{2 s+1}\right)$ we mean

$$
B_{2 s+1}\left(n_{1}, \ldots, n_{s} ; n_{s+1}, \ldots, n_{2 s} ; n_{2 s+1}\right)=G_{2 s+1}\left[K_{n_{1}}, \ldots, K_{n_{2 s+1}}\right],
$$

where the ordering of the vertices of $G_{2 s+1}$ is $V\left(G_{2 s+1}\right)=\left\{v_{1}, \ldots, v_{s}, w_{1}, \ldots, w_{s}, v_{s+1}\right\}$, (see Figure 5). 
Remark 3.2. For every positive integers $s$ and $n_{1}, \ldots, n_{2 s+1}$, one can easily see that

$$
B_{2 s}\left(n_{1}, \ldots, n_{s} ; n_{s+1}, \ldots, n_{2 s}\right) \cong B_{2 s}\left(n_{s+1}, \ldots, n_{2 s} ; n_{1}, \ldots, n_{s}\right),
$$

and

$$
B_{2 s+1}\left(n_{1}, \ldots, n_{s} ; n_{s+1}, \ldots, n_{2 s} ; n_{2 s+1}\right) \cong B_{2 s+1}\left(n_{s+1}, \ldots, n_{2 s} ; n_{1}, \ldots, n_{s} ; n_{2 s+1}\right) .
$$

For avoiding the repeating, using the dictionary ordering on $\left(n_{1}, \ldots, n_{s}\right)$ and $\left(n_{s+1}, \ldots\right.$, $\left.n_{2 s}\right)$ we just cite one of the graphs $B_{2 s}\left(n_{1}, \ldots, n_{s} ; n_{s+1}, \ldots, n_{2 s}\right)$ or $B_{2 s}\left(n_{s+1}, \ldots, n_{2 s}\right.$; $\left.n_{1}, \ldots, n_{s}\right)$ in our characterization. Similarly for the graphs $B_{2 s+1}\left(n_{1}, \ldots, n_{s} ; n_{s+1}, \ldots\right.$, $\left.n_{2 s} ; n_{2 s+1}\right)$ and $B_{2 s+1}\left(n_{s+1}, \ldots, n_{2 s} ; n_{1}, \ldots, n_{s} ; n_{2 s+1}\right)$ we only consider one of them. For example since by dictionary ordering $(4,3,2) \geq(4,3,1)$ we use $B_{6}(4,3,2 ; 4,3,1)$ instead of $B_{6}(4,3,1 ; 4,3,2)$. As another example we use $B_{7}(5,3,2 ; 5,2,4 ; 8)$ instead of $B_{7}(5,2,4 ; 5,3,2 ; 8)$, since $(5,3,2) \geq(5,2,4)$.

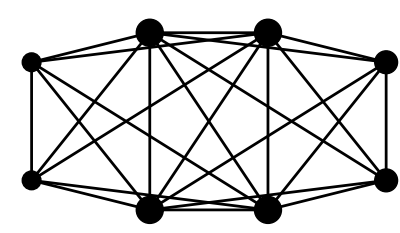

$B_{3}(2 ; 2 ; 4)$

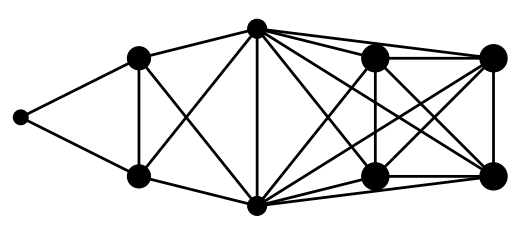

$B_{4}(1,2 ; 4,2)$

Figure 5: The graphs $B_{3}(2 ; 2 ; 4)$ and $B_{4}(1,2 ; 4,2)$.

The following theorem is the main result of [9].

Theorem 3.3. [9] Graph $G$ has the property $\lambda_{3}<0$ if and only if $G$ is an induced subgraph of one of the following graphs:

1. $B_{4}(3,2 ; 2, r)$,

2. $B_{5}(1, r ; 2,3 ; 1)$,

3. $B_{5}(r, 1 ; 2,3 ; 1)$,

4. $B_{5}(3,2 ; 2,1 ; r)$,

5. $B_{5}(r, 2 ; 1,2 ; 2)$,

6. $B_{6}(r, 1, s ; 1,2,2)$,

7. $B_{6}(2,1, r ; 2,1, s)$,

8. $B_{6}(1,2,2 ; 1, r, 1)$,

9. $B_{6}(2,2,1 ; 1,1, r)$,

10. $B_{7}(2,1,1 ; 2,1,1 ; r)$, 
11. $B_{7}(r, 1,2 ; 1, s, 1 ; 1)$,

12. $B_{7}(r, 1,1 ; 1,1,2 ; 1)$,

13. $B_{7}(2,2,1 ; 1, r, 1 ; s)$,

14. $B_{8}(r, 1,1, s ; 1,1, t, 1)$,

15. $B_{9}(1, r, 1,1 ; 1, s, 1,1 ; t)$,

where $r, s$ and $t$ are some positive integers or $G$ is an induced subgraph of one of the 323 graphs with 12 vertices belonging respectively to the classes $B_{4}$ (10 graphs), $B_{5}$ (25 graphs), $B_{6}$ (69 graphs), $B_{7}$ (74 graphs), $B_{8}$ (80 graphs), $B_{9}$ (40 graphs), $B_{10}$ (20 graphs), $B_{11}$ (4 graphs) and $B_{12}$ (1 graph).

Now we give a nicer characterization for graphs with $\lambda_{3}<0$. Note that the Petrović's result shows that any graph with exactly two non-negative eigenvalues is an induced subgraph of one the graphs described by Theorem 3.3. Since finding the structure of induced subgraphs of a graph is complicated, it is better to find the exact structure of all graphs with $\lambda_{3}<0$. In sequel we find this structure. To find our characterization, first we note that by Theorem 2.20 every graph with exactly two non-negative eigenvalues is isomorphic to $G_{s}\left[K_{t_{1}}, \ldots, K_{t_{s}}\right]$ for some positive integers $t_{1}, \ldots, t_{s}$, where $2 \leq s \leq 12$. In this section we find all values of $t_{1}, \ldots, t_{s}$ such that $G_{s}\left[K_{t_{1}}, \ldots, K_{t_{s}}\right]$ has exactly two non-negative eigenvalues. In other words we solve the converse of Theorem 2.20. We note that by Remark 2.6 it suffices to find all connected graphs with $\lambda_{1}>0, \lambda_{2}>0$ and $\lambda_{3}<0$. In other words we find all connected graphs $G_{s}\left[K_{t_{1}}, \ldots, K_{t_{s}}\right]$ such that $3 \leq s \leq 12$ and $\lambda_{1}>0, \lambda_{2}>0$ and $\lambda_{3}<0$. We obtain these graphs in ten theorems (for every $s, 3 \leq s \leq 12$, we consider a theorem). First we prove the case $s=3$. Since the cases $s=4, \ldots, s=9$ similarly are proved, we just prove the case $s=6$. In addition the proofs of the cases $s=10,11,12$ are similar and we only prove the case $s=10$. Our proofs are based on three theorems, Theorem 2.21 for computing the characteristic polynomials of $G_{s}\left[K_{t_{1}}, \ldots, K_{t_{s}}\right]$, Descartes' Sign Rule for polynomials and Interlacing Theorem 2.1. Since $G_{s}\left[K_{t_{1}}, \ldots, K_{t_{s}}\right]=B_{s}\left(t_{1}, \ldots, t_{s}\right)$, in sequel we use $B_{s}\left(t_{1}, \ldots, t_{s}\right)$ instead of $G_{s}\left[K_{t_{1}}, \ldots, K_{t_{s}}\right]$.

Theorem 3.4. Let $G=B_{3}(a ; b ; c)$, where $a, b, c$ are some positive integers. Then $\lambda_{2}(G)>$ 0 and $\lambda_{3}(G)<0$ if and only if $a b \neq 1$.

Proof. Let $g(\lambda)=P\left(B_{3}(a ; b ; c), \lambda\right)$. By Theorem 2.21 we obtain that

$$
g(\lambda)=(\lambda+1)^{a+b+c-3} f(\lambda),
$$

where

$f(\lambda)=\lambda^{3}-(a+b+c-3) \lambda^{2}+(a b-2 a-2 b-2 c+3) \lambda+a c(b-1)+(a-1)(b+c-1)$.

If $a b=1$, that is $a=b=1$, then $g(\lambda)=\lambda(\lambda+1)^{c-1}\left(\lambda^{2}-(c-1) \lambda-2 c\right)$. This shows that $\lambda_{1}(G)>0$ and $\lambda_{2}(G)=0$. Now suppose that $a b \geq 2$. Let $z_{1} \geq z_{2} \geq z_{3}$ be all roots of $f$. Hence $f(\lambda)=\left(\lambda-z_{1}\right)\left(\lambda-z_{2}\right)\left(\lambda-z_{3}\right)$. Therefore $z_{1}+z_{2}+z_{3}=a+b+c-3>0$ and $z_{1} z_{2} z_{3}=-f(0)=-(a c(b-1)+(a-1)(b+c-1))<0$. These equalities show that $z_{1}>0, z_{2}>0$ and $z_{3}<0$. On the other hand by the Equation (3.1), the eigenvalues 
of $B_{3}(a ; b ; c)$ are $z_{1}, z_{2}, z_{3},-1, \ldots,-1$ (the multiplicity of -1 is $a+b+c-3$ ). Hence $\lambda_{1}(G)=z_{1}>0, \lambda_{2}(G)=z_{2}>0$ and $\lambda_{3}(G)=\max \left\{z_{3},-1\right\}<0$. The proof is complete.

Theorem 3.5. Let $G=B_{4}\left(a_{1}, a_{2} ; a_{3}, a_{4}\right)$, where $a_{1}, a_{2}, a_{3}, a_{4}$ are some positive integers. Then $\lambda_{2}(G)>0$ and $\lambda_{3}(G)<0$ if and only if $G$ is isomorphic to one of the following graphs:

1. $B_{4}(a, b ; 1, d), B_{4}(a, x ; y, 1), B_{4}(a, 1 ; c, 1), B_{4}(a, 1 ; w, x)$,

2. $B_{4}(a, 1 ; x, d), \quad B_{4}(w, b ; x, 1), \quad B_{4}(w, x ; y, d), \quad B_{4}(x, b ; y, d)$,

3. 25 specific graphs: 5 graphs of order 10, 10 graphs of order 11, and 10 graphs of order 12 ,

where $a, b, c, d, x, y, w$ are some positive integers such that $x \leq 2, y \leq 2$ and $w \leq 3$.

Theorem 3.6. Let $G=B_{5}\left(a_{1}, a_{2} ; a_{3}, a_{4} ; a_{5}\right)$, where $a_{1}, a_{2}, a_{3}, a_{4}, a_{5}$ are some positive integers. Then $\lambda_{2}(G)>0$ and $\lambda_{3}(G)<0$ if and only if $G$ is isomorphic to one of the following graphs:

1. $B_{5}(a, w ; 1,1 ; 1), B_{5}(a, x ; 1, d ; 1), B_{5}(a, x ; 1, y ; z), \quad B_{5}(a, x ; 1,1 ; e)$,

2. $B_{5}(a, 1 ; c, 1 ; e), B_{5}(a, 1 ; x, w ; 1), B_{5}(a, 1 ; x, y ; e), B_{5}(a, 1 ; 1, d ; e)$,

3. $B_{5}(w, x ; y, 1 ; e), B_{5}(x, b ; 1,1 ; 1), \quad B_{5}(x, w ; 1, d ; 1), \quad B_{5}(x, w ; 1,1 ; e)$,

4. $B_{5}(1, b ; 1, d ; 1), B_{5}(1, b ; 1, x ; y), \quad B_{5}(1, x ; 1, y ; e)$,

5. 63 specific graphs: 13 graphs of order 10, 25 graphs of order 11, and 25 graphs of order 12 ,

where $a, b, c, d, e, x, y, z, w$ are some positive integers such that $x \leq 2, y \leq 2, z \leq 2$ and $w \leq 3$.

Theorem 3.7. Let $G=B_{6}\left(a_{1}, a_{2}, a_{3} ; a_{4}, a_{5}, a_{6}\right)$, where $a_{1}, \ldots, a_{6}$ are some positive integers. Then $\lambda_{2}(G)>0$ and $\lambda_{3}(G)<0$ if and only if $G$ is isomorphic to one of the following graphs:

1. $B_{6}(a, x, c ; 1,1,1), B_{6}(a, 1, c ; 1, e, 1), \quad B_{6}(a, 1, c ; 1, x, y), \quad B_{6}(a, 1, c ; 1,1, f)$,

2. $B_{6}(a, 1,1 ; x, e, 1), B_{6}(x, b, 1 ; y, 1,1), B_{6}(x, y, 1 ; 1, e, 1)$,

3. $B_{6}(x, y, 1 ; 1,1, f), \quad B_{6}(x, 1, c ; y, 1, f), \quad B_{6}(1, b, x ; 1,1,1)$,

4. $B_{6}(1, b, 1 ; 1, e, 1), \quad B_{6}(1, b, 1 ; 1, x, y), \quad B_{6}(1, x, y ; 1,1, f)$,

5. 145 specific graphs: 22 graphs of order 10, 54 graphs of order 11, and 69 graphs of order 12 ,

where $a, b, c, d, e, f, x, y$ are some positive integers such that $x \leq 2$ and $y \leq 2$. 
Proof. Let $\Omega_{6}$ be the set of all 13 above types of graphs. In other words,

$\Omega_{6}=\left\{B_{6}(a, x, c ; 1,1,1), B_{6}(a, 1, c ; 1, e, 1), \ldots, B_{6}(1, b, 1 ; 1, x, y), B_{6}(1, x, y ; 1,1, f)\right\}$,

where $a, b, c, d, e, f$ are arbitrary positive integers and $x, y \in\{1,2\}$. First we prove that every graph of $\Omega_{6}$ has positive second largest eigenvalue and negative third largest eigenvalue. For instance we show that for any positive integers $a, c$ and $f, \lambda_{2}\left(B_{6}(a, 1, c ; 1,1, f)\right)>0$ and $\lambda_{3}\left(B_{6}(a, 1, c ; 1,1, f)\right)<0$. The others are proved similarly.

First we note that $G_{6}$ is an induced subgraph of $B_{6}(a, 1, c ; 1,1, f)$. Thus by Interlacing Theorem 2.1, $\lambda_{2}\left(B_{6}(a, 1, c ; 1,1, f)\right) \geq \lambda_{2}\left(G_{6}\right)>0$. On the other hand, if $m=$ $\max \{a, c, f\}$, then $B_{6}(a, 1, c ; 1,1, f)$ is an induced subgraph of $B_{6}(m, 1, m ; 1,1, m)$. So by Interlacing Theorem $2.1, \lambda_{3}\left(B_{6}(a, 1, c ; 1,1, f)\right) \leq \lambda_{3}\left(B_{6}(m, 1, m ; 1,1, m)\right)$. Thus to show that the inequality $\lambda_{3}\left(B_{6}(a, 1, c ; 1,1, f)\right)<0$, it is sufficient to prove that $\lambda_{3}\left(B_{6}(m\right.$, $1, m ; 1,1, m))<0$. Now we show that for every positive integer $m, \lambda_{3}\left(B_{6}(m, 1, m ; 1,1\right.$, $m))<0$.

Let $M$ and $m$ be two positive integers. If $M \geq m$, then by Interlacing Theorem 2.1, $\lambda_{3}\left(B_{6}(M, 1, M ; 1,1, M)\right) \geq \lambda_{3}\left(B_{6}(m, 1, m ; 1,1, m)\right)$. This shows that if for $m \geq 12$, $\lambda_{3}\left(B_{6}(m, 1, m ; 1,1, m)\right)<0$, then for all $m, \lambda_{3}\left(B_{6}(m, 1, m ; 1,1, m)\right)<0$. Hence suppose that $m \geq 12$. By Theorem 2.21 we can obtain the characteristic polynomial of $B_{6}(m, 1, m ; 1,1, m)$. Let $\Phi_{m}(\lambda)=P\left(B_{6}(m, 1, m ; 1,1, m), \lambda\right)$. By Theorem 2.21

$$
\Phi_{m}(\lambda)=(\lambda+1)^{3 m-3} \Psi_{m}(\lambda),
$$

where

$$
\begin{aligned}
\Psi_{m}(\lambda)= & 3 m+\left(6 m^{2}+7 m-1\right) \lambda+\left(2 m^{3}+12 m^{2}-m-3\right) \lambda^{2}+ \\
& \left(m^{3}+7 m^{2}-14 m-2\right) \lambda^{3}+\left(m^{2}-12 m+2\right) \lambda^{4}+(3-3 m) \lambda^{5}+\lambda^{6} .
\end{aligned}
$$

Since $m \geq 12$, all coefficients of $\Psi_{m}(\lambda)$ are positive except the coefficient of $\lambda^{5}$. In fact the coefficient of $\lambda^{5}$ is negative. Now by Descartes' Sign Rule we conclude that the number of positive roots of $\Psi_{m}(\lambda)$ is 0 or 2 and the number of negative roots is 0 or 2 or 4 . Since $\Psi_{m}(0)=3 m \neq 0$, every root of $\Psi_{m}(\lambda)$ is non-zero. On the other hand by Equation (3.2) the roots of $\Psi_{m}(\lambda)$ with many numbers -1 are the eigenvalues of $B_{6}(m, 1, m ; 1,1, m)$. Hence every root of $\Psi_{m}(\lambda)$ is real. Since $B_{6}(1,1,1 ; 1,1,1) \cong G_{6}$ is an induced subgraph of $B_{6}(m, 1, m ; 1,1, m)$, by Interlacing Theorem 2.1 and Lemma 2.16 we find that $\lambda_{1}\left(B_{6}(m, 1, m ; 1,1, m)\right) \geq \lambda_{1}\left(G_{6}\right)>0$ and $\lambda_{2}\left(B_{6}(m, 1, m ; 1,1, m)\right) \geq \lambda_{2}\left(G_{6}\right)>0$. Therefore by Equation (3.2), $\lambda_{1}\left(B_{6}(m, 1, m ; 1,1, m)\right)$ and $\lambda_{2}\left(B_{6}(m, 1, m ; 1,1, m)\right)$ are two roots of $\Psi_{m}(\lambda)$. Hence $\Psi_{m}(\lambda)$ has exactly two positive roots. Since the degree of $\Psi_{m}(\lambda)$ is six and $\Psi_{m}(\lambda)$ has exactly two positive roots and $\Psi_{m}(0) \neq 0$, the number of negative roots of $\Psi_{m}(\lambda)$ is four. Therefore by Equation (3.2), $B_{6}(m, 1, m ; 1,1, m)$ has exactly two positive eigenvalues and $3 m+1$ negative eigenvalues. This shows that $\lambda_{3}\left(B_{6}(m, 1, m ; 1,1, m)\right)<0$. Now we prove the necessity.

Claim 1. Let $H=B_{6}\left(a^{\prime}, b^{\prime}, c^{\prime} ; d^{\prime}, e^{\prime}, f^{\prime}\right)$ be a graph with at least 19 vertices, that is $a^{\prime}+\cdots+f^{\prime} \geq 19$. If $H \notin \Omega_{6}$, then one of the graphs $H_{1}=B_{6}\left(a^{\prime}-1, b^{\prime}, c^{\prime} ; d^{\prime}, e^{\prime}, f^{\prime}\right)$ or $H_{2}=B_{6}\left(a^{\prime}, b^{\prime}-1, c^{\prime} ; d^{\prime}, e^{\prime}, f^{\prime}\right)$ or $H_{3}=B_{6}\left(a^{\prime}, b^{\prime}, c^{\prime}-1 ; d^{\prime}, e^{\prime}, f^{\prime}\right)$ or $H_{4}=B_{6}\left(a^{\prime}, b^{\prime}, c^{\prime} ;\right.$ $\left.d^{\prime}-1, e^{\prime}, f^{\prime}\right)$ or $H_{5}=B_{6}\left(a^{\prime}, b^{\prime}, c^{\prime} ; d^{\prime}, e^{\prime}-1, f^{\prime}\right)$ or $B_{6}\left(a^{\prime}, b^{\prime}, c^{\prime} ; d^{\prime}, e^{\prime}, f^{\prime}-1\right)$ is not in $\Omega_{6}$. Note that these graphs are all induced subgraphs of $H$ of order $|V(H)|-1$. 
Proof of Claim 1. Suppose that $H \notin \Omega_{6}$. By contradiction assume that all graphs $H_{1}, \ldots, H_{6}$ are in $\Omega_{6}$. Now we consider $H_{1}$. If $H_{1}=B_{6}(a, x, c ; 1,1,1)$ for some positive integers $a, c$ and $x \leq 2$, then $H \in \Omega_{6}$, a contradiction. If $H_{1}=B_{6}(a, 1, c ; 1, e, 1)$, for some positive integers $a, c$ and $e$, then $H \in \Omega_{6}$, a contradiction. Similarly one can see that $H_{1} \neq$ $B_{6}(a, 1, c ; 1, x, y), B_{6}(a, 1, c ; 1,1, f), B_{6}(a, 1,1 ; x, e, 1)$. So $H_{1}=B_{6}(x, b, 1 ; y, 1,1)$ or $B_{6}(x, y, 1 ; 1, e, 1)$ or $B_{6}(x, y, 1 ; 1,1, f)$ or $B_{6}(x, 1, c ; y, 1, f)$ or $B_{6}(1, b, x ; 1,1,1)$ or $B_{6}(1, b, 1 ; 1, e, 1)$ or $B_{6}(1, b, 1 ; 1, x, y)$ or $B_{6}(1, x, y ; 1,1, f)$, for some positive integers $b, c, e, f$ and $x, y \leq 2$. Since $x \leq 2$, we find that $a^{\prime}-1 \leq 2$. Thus $a^{\prime} \leq 3$. Similarly if $H_{2}, \ldots, H_{6} \in \bar{\Omega}_{6}$, we obtain that $b^{\prime}, \ldots, f^{\prime} \leq 3$. Therefore $a^{\prime}+\cdots+f^{\prime} \leq 18$, a contradiction. Thus the claim is proved.

Claim 2. Let $K=B_{6}\left(a^{\prime \prime}, b^{\prime \prime}, c^{\prime \prime} ; d^{\prime \prime}, e^{\prime \prime}, f^{\prime \prime}\right)$ be a graph with at least 13 vertices. If $K \notin \Omega_{6}$, then $\lambda_{3}(K) \geq 0$.

Proof of Claim 2. Assume that $K \notin \Omega_{6}$. We prove the claim by induction on $n=$ $|V(K)|$. By computer one can check the validity for $n=13, \ldots, 18$. Hence let $n \geq 19$. By Claim $1, K$ has an induced subgraph, say $L$, of order $n-1$ such that $L \notin \Omega_{6}$. Since $n-1 \geq 18$, by the induction hypothesis $\lambda_{3}(L) \geq 0$. Thus by Interlacing Theorem 2.1, $\lambda_{3}(K) \geq \lambda_{3}(L) \geq 0$. Thus the claim is proved.

Now let $W=B_{6}\left(a^{\prime \prime \prime}, b^{\prime \prime \prime}, c^{\prime \prime \prime} ; d^{\prime \prime \prime}, e^{\prime \prime \prime}, f^{\prime \prime \prime}\right)$ be a graph of order $n$. Assume that $\lambda_{2}(W)>0$ and $\lambda_{3}(W)<0$. If $W \notin \Omega_{6}$, then by Claim $2, n \leq 12$. By computer we find that there are only 145 graphs with this property. More precisely there are 22 graphs of order 10, 54 graphs of order 11 and 69 graphs of order 12 such that they are not in $\Omega_{6}$ while their second eigenvalues are positive and third eigenvalues are negative. The proof is complete.

Theorem 3.8. Let $G=B_{7}\left(a_{1}, a_{2}, a_{3} ; a_{4}, a_{5}, a_{6} ; a_{7}\right)$, where $a_{1}, \ldots, a_{7}$ are some positive integers. Then $\lambda_{2}(G)>0$ and $\lambda_{3}(G)<0$ if and only if $G$ is isomorphic to one of the following graphs:

1. $B_{7}(a, 1, x ; 1, e, 1 ; 1), B_{7}(a, 1,1 ; 1, e, 1 ; g), \quad B_{7}(a, 1,1 ; 1,1, x ; 1)$,

2. $B_{7}(x, y, 1 ; 1, e, 1 ; g), B_{7}(x, 1,1 ; y, 1,1 ; g), B_{7}(1, b, x ; 1,1,1 ; g)$,

3. $B_{7}(1, b, 1 ; 1, e, 1 ; g), B_{7}(1,1, c ; 1,1, f ; 1)$,

4. 143 specific graphs: 18 graphs of order 10, 52 graphs of order 11, and 73 graphs of order 12 ,

where $a, b, c, d, e, f, g, x, y$ are some positive integers such that $x \leq 2$ and $y \leq 2$.

Theorem 3.9. Let $G=B_{8}\left(a_{1}, a_{2}, a_{3}, a_{4} ; a_{5}, a_{6}, a_{7}, a_{8}\right)$, where $a_{1}, \ldots, a_{8}$ are some positive integers. Then $\lambda_{2}(G)>0$ and $\lambda_{3}(G)<0$ if and only if $G$ is isomorphic to one of the following graphs:

1. $B_{8}(a, 1,1, d ; 1,1, g, 1), B_{8}(1, b, 1,1 ; 1, f, 1,1)$,

2. 134 specific graphs: 12 graphs of order 10, 42 graphs of order 11, and 80 graphs of order 12 ,

where $a, b, d, f, g$ are some positive integers. 
Theorem 3.10. Let $G=B_{9}\left(a_{1}, a_{2}, a_{3}, a_{4} ; a_{5}, a_{6}, a_{7}, a_{8} ; a_{9}\right)$, where $a_{1}, \ldots, a_{9}$ are some positive integers. Then $\lambda_{2}(G)>0$ and $\lambda_{3}(G)<0$ if and only if $G$ is isomorphic to one of the following graphs:

1. $B_{9}(1, b, 1,1 ; 1, f, 1,1 ; k)$,

2. 59 specific graphs: 3 graphs of order 10, 17 graphs of order 11, and 39 graphs of order 12 ,

where $b, f, k$ are some positive integers.

Remark 3.11. The complete list of the mentioned 25 graphs in Theorem 3.5, 63 graphs in Theorem 3.6, 145 graphs in Theorem 3.7, 143 graphs in Theorem 3.8, 134 graphs in Theorem 3.9 and 59 graphs in Theorem 3.10 can be obtained from the author upon request.

Theorem 3.12. Let $G=B_{10}\left(a_{1}, a_{2}, a_{3}, a_{4}, a_{5} ; a_{6}, a_{7}, a_{8}, a_{9}, a_{10}\right)$, where $a_{1}, \ldots, a_{10}$ are some positive integers. Then $\lambda_{2}(G)>0$ and $\lambda_{3}(G)<0$ if and only if $G$ is isomorphic to one of the following 26 graphs:

1. $B_{10}(1,1,1,1,1 ; 1,1,1,1,1)$,

2. $B_{10}(1,1,1,1,2 ; 1,1,1,1,1), B_{10}(1,1,1,2,1 ; 1,1,1,1,1)$,

3. $B_{10}(1,1,2,1,1 ; 1,1,1,1,1), B_{10}(1,2,1,1,1 ; 1,1,1,1,1)$,

4. $B_{10}(2,1,1,1,1 ; 1,1,1,1,1)$,

5. $B_{10}(1,1,1,1,3 ; 1,1,1,1,1), B_{10}(1,1,1,2,1 ; 1,1,1,1,2)$,

6. $B_{10}(1,1,1,2,1 ; 1,1,1,2,1), B_{10}(1,1,1,2,2 ; 1,1,1,1,1)$,

7. $B_{10}(1,1,1,3,1 ; 1,1,1,1,1), B_{10}(1,1,2,1,1 ; 1,1,1,1,2)$,

8. $B_{10}(1,1,2,2,1 ; 1,1,1,1,1), B_{10}(1,1,3,1,1 ; 1,1,1,1,1)$,

9. $B_{10}(1,2,1,1,1 ; 1,1,2,1,1), B_{10}(1,2,1,1,1 ; 1,2,1,1,1)$,

10. $B_{10}(1,2,1,1,2 ; 1,1,1,1,1), B_{10}(1,2,2,1,1 ; 1,1,1,1,1)$,

11. $B_{10}(1,3,1,1,1 ; 1,1,1,1,1), B_{10}(2,1,1,1,1 ; 1,1,1,2,1)$,

12. $B_{10}(2,1,1,1,1 ; 1,1,2,1,1), B_{10}(2,1,1,1,1 ; 2,1,1,1,1)$,

13. $B_{10}(2,1,1,1,2 ; 1,1,1,1,1), B_{10}(2,1,1,2,1 ; 1,1,1,1,1)$,

14. $B_{10}(2,2,1,1,1 ; 1,1,1,1,1), B_{10}(3,1,1,1,1 ; 1,1,1,1,1)$.

Proof. One can see that all of the above graphs have positive second largest eigenvalue and negative third largest eigenvalue. Now we prove the necessity. Let $G=B_{10}\left(a_{1}, \ldots, a_{5} ; a_{6}\right.$, $\left.\ldots, a_{10}\right)$ such that $\lambda_{2}(G)>0$ and $\lambda_{3}(G)<0$. We show that for $i=1, \ldots, 10, a_{i} \leq 3$. For example by contradiction suppose that $a_{1} \geq 4$. Thus $H=B_{10}(4,1,1,1,1 ; 1,1,1,1,1)$ is an induced subgraph of $G$. So by Interlacing Theorem 2.1, $\lambda_{3}(G) \geq \lambda_{3}(H)$. On the other hand $\lambda_{3}(H)>0$, a contradiction. Similarly we obtain $a_{2}, \ldots, a_{10} \leq 3$. Also one can 
see that at most one of the numbers $a_{1}, \ldots, a_{10}$ is 3 . For example if $a_{1}=3$ and $a_{2}=3$, then $K=B_{10}(3,3,1,1,1 ; 1,1,1,1,1)$ is an induced subgraph of $G$. So by Interlacing Theorem 2.1, $\lambda_{3}(G) \geq \lambda_{3}(K)$ while $\lambda_{3}(K)>0$, a contradiction. Since $a_{1}, \ldots, a_{10} \leq 3$ and at most one of them is $3, a_{1}+\cdots+a_{10} \leq 21$. Thus the order of $G$ is at most 21 . Now by computer one can check the result.

Theorem 3.13. Let $G=B_{11}\left(a_{1}, a_{2}, a_{3}, a_{4}, a_{5} ; a_{6}, a_{7}, a_{8}, a_{9}, a_{10} ; a_{11}\right)$, where $a_{1}, \ldots, a_{11}$ are some positive integers. Then $\lambda_{2}(G)>0$ and $\lambda_{3}(G)<0$ if and only if $G$ is isomorphic to one of the following 5 graphs:

$$
\begin{aligned}
& \text { 1. } B_{11}(1,1,1,1,1 ; 1,1,1,1,1 ; 1), \\
& \text { 2. } B_{11}(1,1,1,1,1 ; 1,1,1,1,1 ; 2), B_{11}(1,1,1,1,2 ; 1,1,1,1,1 ; 1), \\
& \text { 3. } B_{11}(1,2,1,1,1 ; 1,1,1,1,1 ; 1), B_{11}(1,1,2,1,1 ; 1,1,1,1,1 ; 1) .
\end{aligned}
$$

Theorem 3.14. Let $G=B_{12}\left(a_{1}, a_{2}, a_{3}, a_{4}, a_{5}, a_{6} ; a_{7}, a_{8}, a_{9}, a_{10}, a_{11}, a_{12}\right)$, where $a_{1}, \ldots$, $a_{12}$ are some positive integers. Then $\lambda_{2}(G)>0$ and $\lambda_{3}(G)<0$ if and only if $G \cong$ $B_{12}(1,1,1,1,1,1 ; 1,1,1,1,1,1)$.

\section{Acknowledgements}

The author is grateful to the referees for their useful comments.

\section{References}

[1] A. Abdollahi, Sh. Janbaz and M. R. Oboudi, Distance between spectra of graphs, Linear Algebra Appl. 466 (2015), 401-408.

[2] A. Abdollahi and M. R. Oboudi, Cospectrality of graphs, Linear Algebra Appl. 451 (2014), $169-181$.

[3] D. M. Cvetković, M. Doob and H. Sachs, Spectra of graphs: Theory and application, Academic Press, Inc., New York-London, 1980.

[4] C. Godsil and G. Royle, Algebraic graph theory, Springer-Verlag, New York, 2001.

[5] M. R. Oboudi, Bipartite graphs with at most six non-zero eigenvalues, Ars Math. Contemp. 11 (2016), 315-325.

[6] M. R. Oboudi, Cospectrality of complete bipartite graphs, Linear Multilinear Algebra 64 (2016), 2491-2497.

[7] M. R. Oboudi, On the third largest eigenvalue of graphs, Linear Algebra Appl. 503 (2016), $164-179$.

[8] M. R. Oboudi, On the difference between the spectral radius and the maximum degree of graphs, to appear.

[9] M. Petrović, Graphs with a small number of nonnegative eigenvalues, Graphs Combin. 15 (1999), 221-232.

[10] J. H. Smith, Symmetry and multiple eigenvalues of graphs, Glasnik Mat. Ser. III 12 (1977), $3-8$. 